\title{
Image-model coupling: application to an ionospheric storm
}

\author{
N. D. Smith ${ }^{1, *}$, D. Pokhotelov ${ }^{2}$, C. N. Mitchell ${ }^{2}$, and C. J. Budd ${ }^{1}$ \\ ${ }^{1}$ Department of Mathematical Sciences, University of Bath, Bath, BA2 7AY, UK \\ ${ }^{2}$ Department of Electronic and Electrical Engineering, University of Bath, Bath, BA2 7AY, UK \\ *Completed while with the Department of Electronic and Electrical Engineering, University of Bath, Bath, BA2 7AY, UK
}

Received: 7 October 2008 - Revised: 16 July 2010 - Accepted: 26 July 2010 - Published: 20 August 2010

\begin{abstract}
Techniques such as tomographic reconstruction may be used to provide images of electron content in the ionosphere. Models are also available which attempt to describe the dominant physical processes operating in the ionosphere, or the statistical relationships between ionospheric variables. It is sensible to try and couple model output to tomographic images with the aim of inferring the values of driver variables which best replicate some description of electron content imaged in the ionosphere, according to some criterion. This is a challenging task. The following describes an attempt to couple an ionospheric model to a tomographic reconstruction of the geomagnetic storm of 20 November 2003, along a latitudal line segment above north America. A simple model was chosen to reduce the number of input drivers that were varied. The investigation illustrates some of the issues involved in image-model coupling. The ability to make scientific deductions depends on the accuracy of the assumptions in the ionospheric model and the accuracy of the tomographic reconstruction. An ensemble technique was used to help assess confidence in the reconstruction.
\end{abstract}

\section{Introduction}

The ionosphere is a complex system with multiple processes operating at different scales. Understanding these processes is of scientific value, and has practical benefit in applications such as communication and navigation. In recent years, different empirical and physical models have been developed to explain how the ionosphere, typically in terms of its plasma content, responds to external stimuli or drivers. The mapping from the space of driver variables to the space of variables describing the ionosphere is expected to be nonlinear, especially for an extreme event such as a geomagnetic storm.

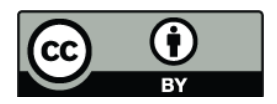

Correspondence to: N. D. Smith (n.smith@bath.ac.uk)
Hence it would be interesting to study the sensitivity of the response to different drivers. This may give further insight into which processes dominate under different conditions. Also, those drivers to which the response is most sensitive may require more accurate or more frequent measurement in future.

One means of investigating the ionosphere, including sensitivity of its response to drivers, is via image-model coupling. This is a challenging task. This paper describes some relevant issues, and presents techniques which may be useful for future developments in image-model coupling. Images of electron density may be obtained, for example, by tomographic reconstruction techniques (Bust and Mitchell, 2008; Pryse et al., 1998). Once a sequence of images is obtained for an ionospheric event, an ionospheric model is then selected, its drivers varied, and the closeness of match between the model output and image sequence calculated. The shape of the matching function gives an indication of sensitivity, for the particular event and subject to the accuracy of the ionospheric model. This approach may also encourage a better appreciation of the limitations and assumptions in the ionospheric model being used. A framework for describing image-model coupling in terms of communication along a discrete channel is presented in (Smith et al., 2009). This paper describes a simple application of these ideas to an extreme event, the geomagnetic storm of 20 November 2003. The matching function is very simple and many of the statistical conditional dependencies between data at successive timesteps are ignored. The features and matching function are described briefly in Sect. 2. The tomographic reconstruction is presented in Sect. 3, and the analysis for an ionospheric model in Sect. 4. Some discussion and conclusions follow in Sects. 5 and 6, respectively.

For reference, Fig. 1 details the variation of variables measured or calculated for 20 November 2003. The Dst index, an indication of geomagnetic activity, shows that during this day the storm peaks at 20:00/21:00 UT. The interplanetary magnetic field (IMF) Bz component shows that the storm,

Published by Copernicus Publications on behalf of the European Geosciences Union and the American Geophysical Union. 

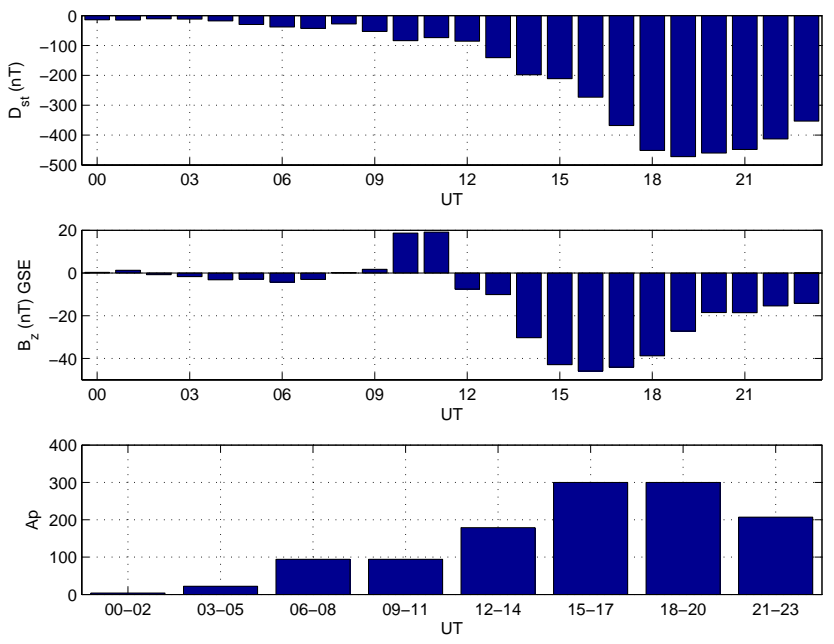

Fig. 1. Hourly measurements for Dst and Bz, and three-hourly measurements for Ap (see OMNIWeb, access: July 2010 and the Acknowledgments).

as expected, occurs during a southward orientation of the IMF (values for the IMF $\mathrm{Bz}$ component are also available at a higher sampling rate than hourly). In addition, the $F_{10.7}$ value for the day was 171.0 (see OMNIWeb, access: July 2010, see also the Acknowledgments).

\section{Calculating features and optimising the match}

It is often convenient to calculate ionospheric data at a discrete set of points on a grid which is uniform in altitude, and geographic latitude and longitude coordinates. The grid points are represented as blackened circles in the sketch of a slice of constant geographic latitude in Fig. 2. Assume the grid is indexed by $(l, m, n)$ where $l \in[1, L]$ is an index for altitude above the Earth's surface, $m \in[1, M]$ for geographic latitude and $n \in[1, N]$ for geographic longitude. The altitude of a grid point is $h_{\mathrm{g}}(l, m, n)$ and the electron density at that point is $N_{\mathrm{g}}(l, m, n)$. The grid points are used to define cells in the manner of Fig. 2 where grid points are cell centres; the exceptions are the "half-cells" at the top and foot of the cell structure. Hence cell centres $h_{\mathrm{c}}(l, m, n)$ are defined with the following altitudes. For all $l, m$,

$$
\begin{aligned}
& h_{\mathrm{c}}(l, m, n)= \\
& \left\{\begin{array}{rll}
\frac{3}{4} h_{\mathrm{g}}(L, m, n)+\frac{1}{4} h_{\mathrm{g}}(L-1, m, n) & \text { if } \quad l=L \\
h_{\mathrm{g}}(l, m, n) & \text { if } \quad 1<l<L, \\
\frac{3}{4} h_{\mathrm{g}}(1, m, n)+\frac{1}{4} h_{\mathrm{g}}(2, m, n) & \text { if } \quad l=1
\end{array}\right.
\end{aligned}
$$

where it is assumed $L>1$. The electron densities at the cell centres are defined such that $N_{\mathrm{c}}(l, m, n)=N_{\mathrm{g}}(l, m, n)$, $\forall l, m, n$. Electron density is assumed uniform in a cell. The cells are contiguous, and each cell has a vertical length

Nonlin. Processes Geophys., 17, 361-369, 2010

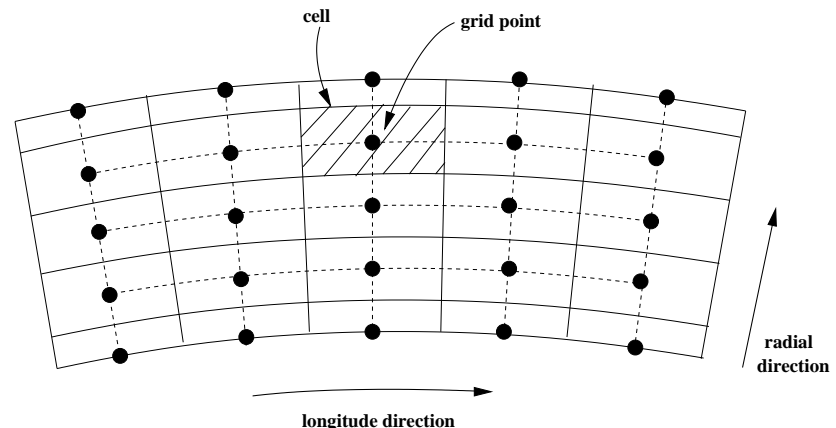

Fig. 2. Sketch showing how grid points are related to cells.

$d(l, m, n)$. The electron content of the ionosphere may be summarised using vertical total electron content (VTEC) or mean ionospheric height (i.e. the height of the centre of mass of the electrons). In the following experiments, VTEC is used.

$\operatorname{VTEC}(m, n)=\sum_{l=1}^{L} d(l, m, n) N_{\mathrm{c}}(l, m, n)$.

The 2-D map may then be assembled into a column vector, for example the $(M N \times 1)$ feature vector,

$z=(\operatorname{VTEC}(1,1), \ldots \operatorname{VTEC}(M, N))^{\top}$.

Assume an image sequence $z(1, l)=\left(z_{1}, \ldots, z_{l}\right)$ obtained by tomographic reconstruction, and output $z^{\prime}(1, l)=\left(z_{1}^{\prime}, \ldots, z_{l}^{\prime}\right)$ obtained by a physical model. Then, giving equal weight to each cell, the unweighted sum square error between the two sequences is,

$f\left(z(1, l), z^{\prime}(1, l)\right)=\sum_{t=1}^{l}\left(z_{t}-z_{t}^{\prime}\right)^{\top}\left(z_{t}-z_{t}^{\prime}\right)$.

Given competing model outputs, the output of closest match is,

$\hat{z}^{\prime}(1, l)=\arg _{z^{\prime}(1, l)} \min f\left(z(1, l), z^{\prime}(1, l)\right)$.

This minimisation is the least sum square error estimate. In the following experiments, each model output sequence was obtained by fixing a subset of driver variables at a vector value $\boldsymbol{u}$. Hence,

$\hat{\boldsymbol{u}}=\arg _{\boldsymbol{u}} \min f(\boldsymbol{z}(1, l), \boldsymbol{u})$,

assuming $\boldsymbol{u} \mapsto \boldsymbol{z}^{\prime}(1, l)$ is injective. In effect, the physical model is being used to "decode" the values of driver variables, assuming the tomographic reconstruction is correct and true. As described more fully in (Smith et al., 2009), $\hat{\boldsymbol{u}}$ may be regarded as the maximum a-posteriori estimate for a simple discrete channel model subject to stationary, additive, indepent and identically distributed (i.i.d.), white Gaussian channel noise. Such a channel model is overly simple for such a complex system as the ionosphere, but permits the derivation of a matching function which is relatively easy to evaluate.

www.nonlin-processes-geophys.net/17/361/2010/ 


\section{Tomographic reconstruction using MIDAS}

MIDAS ("Multi-Instrument Data Analysis System") version 3 (see Appendix A, and a previous version of the software described in Mitchell and Spencer, 2003) was used to tomographically reconstruct electron densities above north America using the total electron content (or slant TEC) along raypaths between satellites and IGS ${ }^{1}$ receiver stations. The reconstruction was conducted hourly from 12:00 UT to 23:00 UT inclusive for 20 November 2003, and over a $(39 \times 6 \times 7)$ structure of grid points (this included altitudes at $90 \mathrm{~km}$, and from $100 \mathrm{~km}$ to $1580 \mathrm{~km}$ inclusive at $40 \mathrm{~km}$ intervals; geographic latitudes from $25^{\circ} \mathrm{N}$ to $50^{\circ} \mathrm{N}$ inclusive at $5^{\circ}$ intervals; geographic longitudes from $130^{\circ} \mathrm{W}$ to $70^{\circ} \mathrm{W}$ inclusive at $10^{\circ}$ intervals). Two sets of data were used; the training set collected from 89 receivers and the evaluation set collected from 82 receivers. The two sets of receivers did not share members, and the receivers were fairly evenly selected from those IGS $^{1}$ receivers available. Locations are detailed in Fig. 3.

The receiver data was first sampled at 5 min intervals over the entire day. At 12:00 UT, the ionosphere was assumed static for $20 \mathrm{~min}$ from 11:50 UT to 12:10 UT (5 frames of data). An estimate for electron content was obtained by seeking those electron densities at each point in the grid which minimised the regularised sum square error of slant TEC as measured by each receiver/transmitter pair. For each pair, the unknown offset relating the phase difference between the two channels of the dual-frequency receiver and slant TEC was also assumed fixed across the $20 \mathrm{~min}$ window, and estimated to minimise the regularised sum square error of slant TEC. The regularised least sum square error problem can be solved by an appropriate quadratic programming solver (here MATLAB ${ }^{2}$ 's quadprog function was used, explicitly enforcing nonnegativity constraints for electron densities, with a suitable tolerance and a limit in the number of iterations, and a fixed nonzero initialisation). The optimisation was then repeated hourly until 23:00 UT inclusive.

Details of the optimisation and regularisation are given briefly in Appendix A. Regularisation involved smoothing the zeroth, first and second-order derivatives of the electron densities in four horizontal spatial directions towards those in the International Reference Ionosphere, 1995 (IRI-95) (Bilitza, 1997); the IRI model was also used in a different though similar approach in Bhuyan and Bhuyan (2007). The amount of regularisation was governed by regularisation parameters $\lambda_{3}^{i} \in\{0,0.0001,0.001,0.01,0.1,1,10,100\}$ for secondorder derivatives, and $\lambda_{k}^{i} \in\{0,0.0001,0.01,1,100\}, k \in\{1,2\}$ for zeroth and first-order derivatives, where larger values of $\lambda_{k}^{i}, k \in\{1,2,3\}$ indicate greater smoothing and $i$ is simply an index for the model being trained. Hence a tomographic reconstruction was obtained for each permutation of

\footnotetext{
${ }^{1}$ http://igscb.jpl.nasa.gov

${ }^{2}$ The MathWorks, http://www.mathworks.com
}

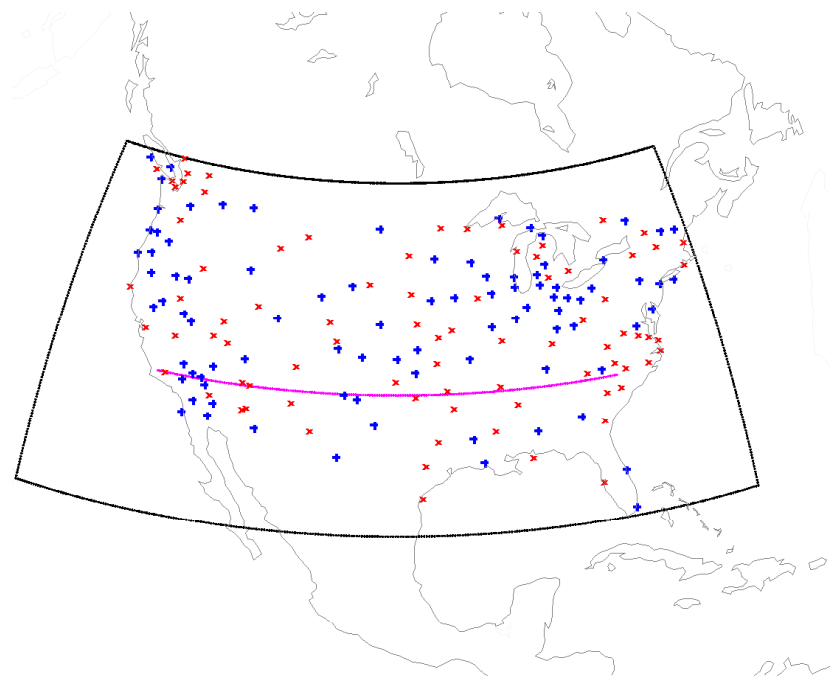

Fig. 3. Locations of IGS ${ }^{1}$ receivers used in training (blue " +") and evaluation (red " $\mathrm{x}$ "); the tomographic reconstruction is delimited by the black box, and the comparison with SAMI2 output is along the central magenta line.

$\left(\lambda_{1}^{i}, \lambda_{2}^{i}, \lambda_{3}^{i}\right)$ except the unregularised solution (i.e. $\left.\lambda_{k}^{i}=0, \forall k\right)$. The ensemble of 199 members was reduced to 185 members by excluding those which contained electron densities less than $-1 \times 10^{9}$ electrons $/ \mathrm{m}^{3}$ (most probably the nonnegativity constraints were violated due to numerical limitations in the constrained optimiser). For each member of the new ensemble, the sum square error of slant TEC for the evaluation set was calculated (again assuming the ionosphere was fixed over a $20 \mathrm{~min}$ window, and that the unknown offsets were also fixed over the time window and were estimated to minimise the sum square error on the evaluation set). The evaluation performance was used in a posterior weighting scheme. The candidate reconstruction for each hour was calculated as the ensemble mean, with confidence provided by the ensemble variance (see Appendix A for further details). However the arithmetic mean of posterior weights was not calculated across all 12 hourly timesteps, but only 9 hourly timesteps. The scaled likelihoods at each timestep were very small and were forced to zero when precision was lost. All scaled likelihoods were set to zero at 18:00, 20:00, and 21:00 UT, so posteriors could not be calculated at those timesteps, or used in calculating the arithmetic mean of posterior weights. At other timesteps, between 1 and 143 (inclusive) members of the ensemble yielded nonzero likelihood and hence nonzero member posterior. The thresholding of member posteriors to zero due to loss in precision is not regarded as problematic, since it may be interpreted as a means of increasing robustness.

To improve the conditioning of the problem and reduce underdeterminacy, a limited set of vertical basis functions were used to represent the vertical electron density profiles. Rather than estimate $(39 \times 6 \times 7)$ electron densities for the full grid 


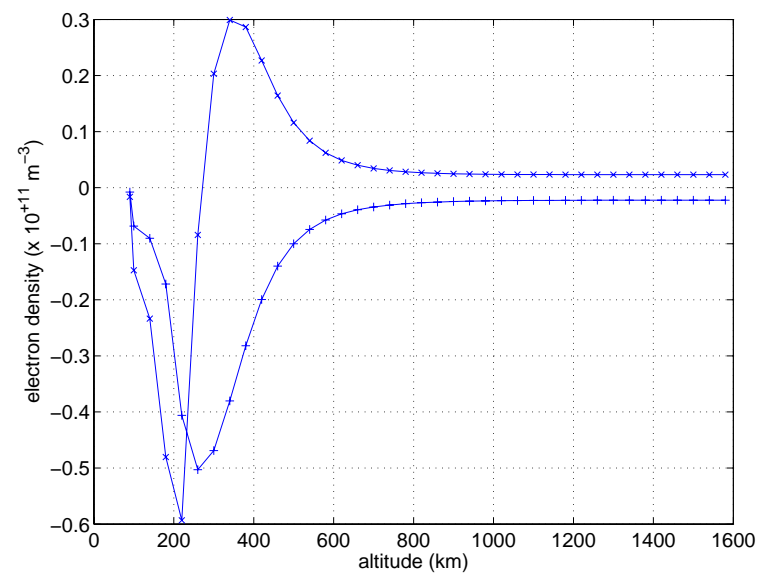

Fig. 4. Vertical basis functions used for the tomographic reconstructions with MIDAS.

structure, only $(n \times 6 \times 7)$ basis function coefficients were estimated, where $n$ is the number of basis functions in the limited set (Spencer and Mitchell, 2007). The basis functions used were those $n$ left singular vectors with largest singular values, calculated from data extracted from IRI-95 (above the reconstruction region, eight times per day for four days during the year 1995).

In the following experiments, we chose $n=2$. The vertical basis functions are plotted in Fig. 4. Vertical profiles were therefore restricted to linear combinations of the basis functions. Admittedly, the basis functions are restrictive and should ideally be optimised dynamically from hour to hour using independent data. However this would further complicate the optimisation problem. For simplicity, the fixed set of vertical basis functions detailed above were always used, and the inability to model high electron densities in the topside, and small-scale features elsewhere, acknowledged.

For a latitudinal line at $35^{\circ} \mathrm{N}$ between $120^{\circ} \mathrm{W}$ to $80^{\circ} \mathrm{W}$ inclusive, the ensemble average VTEC is plotted in Fig. 5 at each $10^{\circ}$ spacing, hourly from 12:00 UT to 23:00 UT inclusive, as a full line. Error bars corresponding to a $95 \%$ confidence level, albeit with a simple Gaussian error model, are also detailed. The error bars show that there is a high degree of variability across the ensemble, without even considering the extra variability expected from varying other parameters such as the number or shape of vertical basis functions, or the size of cells. The variability is in part due to the lack of constraints via horizontal basis functions, and indicates the significant challenge of such underdetermined inverse problems. There is highest variability towards the eastern coast of north America. The "fixed ionosphere" assumption over 20 min may be problematic during the stormy periods.

The onset of the storm can be monitored by viewing the orientation of the IMF and the Dst index (see Fig. 1). Different physical processes can be used to help explain the high plasma densities at midlatitudes during the geomegnetic storm, e.g. Mannucci et al. (2005); Tsurutani et al. (2007); Foster et al. (2005). High plasma densities are probably caused by the interaction of both equatorial/low latitude processes, such as a superfountain effect, and subauroral/high latitude processes such as electron precipitation and Joule heating. Neutral winds and electric fields can lift plasma upwards along field lines, thereby providing mechanisms to increase vertical total electron content at high and midlatitude locations.

\section{Coupling with SAMI2}

SAMI2 ("Sami2 is Another Model of the Ionosphere") version 0.98 (SAMI2, access: November 2007; Huba et al., 2000) is a physics-based model of the ionosphere which solves "first principles equations" describing ionospheric plasma. SAMI2 is able to model plasma along field lines, but constrained within a slice of constant geomagnetic longitude, i.e. a flux tube. Here, there were 10 different field lines within a slice where the maximum altitudes of the highest and lowest field lines were fixed at $10000.0 \mathrm{~km}$ and $100.0 \mathrm{~km}$ respectively, and the base of field lines was fixed at $90.0 \mathrm{~km}$. Densities were calculated at 101 points spaced along each field line. In total, eight different slices were chosen, where the slices were anchored to intersection points defined at $100.0 \mathrm{~km}$ altitude, $40^{\circ} \mathrm{N}$ geographic latitude, and geographic longitude from $60.0^{\circ} \mathrm{W}$ to $130.0^{\circ} \mathrm{W}$ inclusive at $10^{\circ}$ intervals. Seven ion species were modelled by SAMI2. Quasineutrality was assumed in this analysis and the sum of the seven ion densities at a point was taken as the local electron density at that point. The optimisation of SAMI2 against MIDAS was restricted to a latitudinal line segment above north America at $35^{\circ} \mathrm{N}$, compromising adequate coverage by SAMI2 with reliability in the MIDAS reconstruction (as indicated by adequate coverage of receivers). In geographic coordinates, the line stretches from $120^{\circ} \mathrm{W}$ to $80^{\circ} \mathrm{W}$ inclusive with grid points at $10^{\circ}$ intervals and altitudes from $100 \mathrm{~km}$ to $1580 \mathrm{~km}$ inclusive in intervals of $40 \mathrm{~km}$, and also at $90 \mathrm{~km}$. The nonuniformly-spaced set of electron density values from SAMI2 were then mapped onto this plane by nearest pixel sampling, where any ties were resolved by precedence in scan order. In the following experiments, each SAMI2 run was started at 00:00 UT and lasted $48 \mathrm{~h}$ (during which SAMI2 does not update its "day of year"). To reduce the effect of transients, hourly output was collected during the appropriate time window between 24 and $48 \mathrm{~h}$ after the start of each run; hence for the earliest time window beginning at 12:00 UT, SAMI2 was allowed to run for a full $36 \mathrm{~h}$ before output was collected. Two of the principal driver variables for SAMI 2 were $F_{10.7}$ and Ap which are respectively measures of solar and geomagnetic activity (Hargreaves, 2003). The 3-monthly average for $F_{10.7}$ was fixed to the current $F_{10.7}$ value. The parameter associated with photoelectron heating was fixed at $3.0 \times 10^{-14} \mathrm{~cm}^{2}$. The sinusoidal 

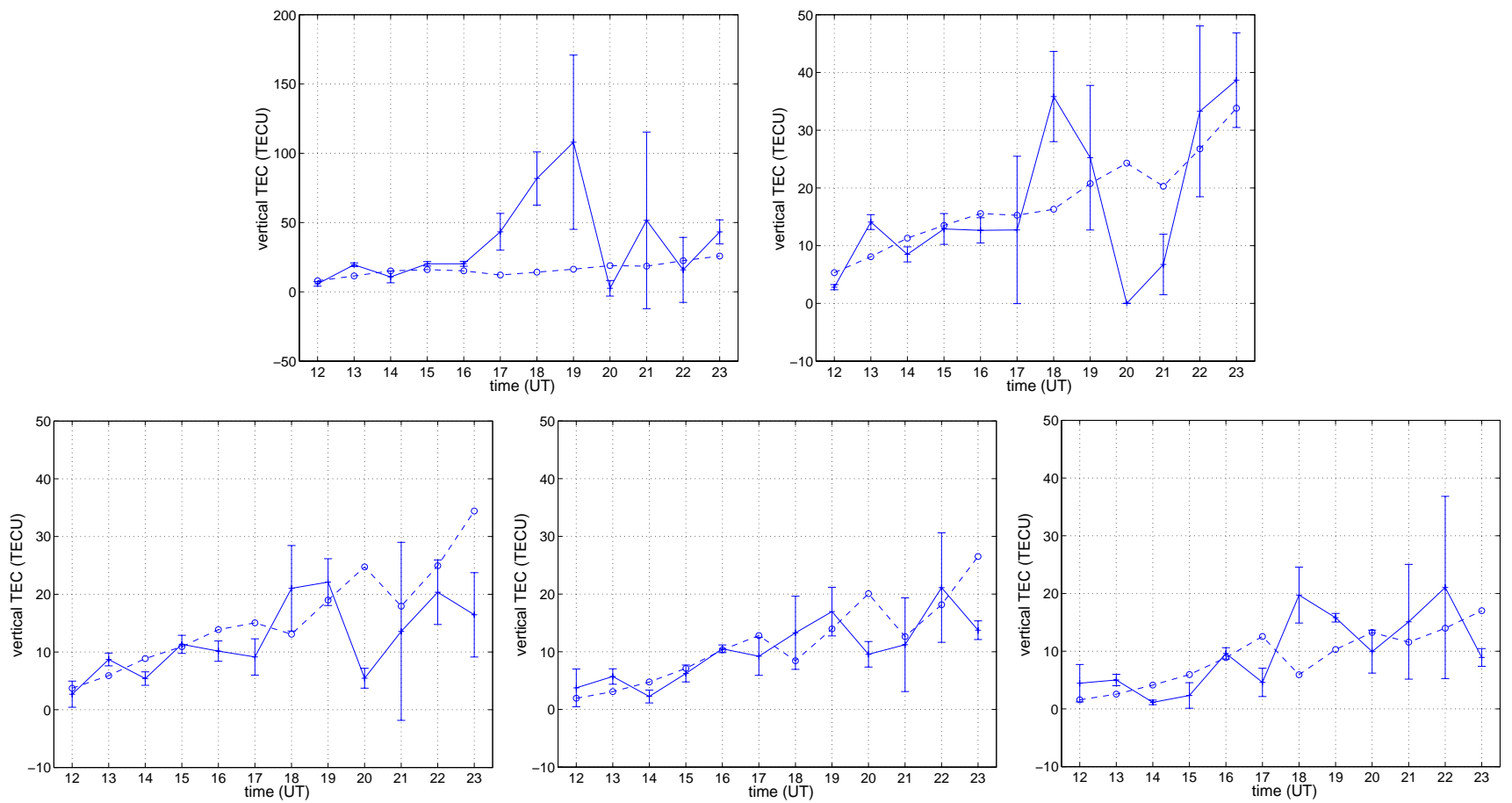

Fig. 5. VTEC profiles at $35^{\circ} \mathrm{N}$ between $120^{\circ} \mathrm{W}$ and $80^{\circ} \mathrm{W}$ inclusive (at $10^{\circ}$ intervals) for 20 November 2003 ; the full line is the ensemble mean from MIDAS with error bars at $95 \%$ confidence level; the dashed line is the optimal match with SAMI $2(1 \mathrm{TECU}=1 \times$ $10^{16}$ electrons $\left./ \mathrm{m}^{2}\right)$.

$\boldsymbol{E} \times \boldsymbol{B}$ drift velocity model was used (Huba et al., 2000), parameterised by $V$ and described in Appendix B. The maximum number of timesteps was set to $20 \times 10^{6}$. All other values which were not directly varied in the following experiments were set to their recommended or default settings; in particular, multiplicative factors for neutral wind speed, $\boldsymbol{E} \times \boldsymbol{B}$ drift velocity, neutral densities corresponding to the 7 positive ion species, and neutral temperature were all kept at unity unless otherwise stated.

In the following investigations, the key driver variables varied for SAMI2 were $F_{10.7}$, Ap, and $V$ which are respectively a measure of solar radiation at $10.7 \mathrm{~cm}$ wavelength, a measure of geomagnetic activity, and the maximum $\boldsymbol{E} \times \boldsymbol{B}$ drift velocity for the sinusoidal drift model (indirectly an indication of electric field strength). These driver variables were varied such that $F_{10.7} \in\{50.0,100.0,150.0,200.0,250.0,300.0\}, \quad \mathrm{Ap} \in$ $\{0,15,80,207,400\}$ which corresponds to $\mathrm{Kp} \in\{0,3,6,8,9\}$, and $V \in\{100.0,200.0,225.0,300.0,375.0,450.0\} \mathrm{ms}^{-1}$. All other driver variables were kept at fixed values unless otherwise stated.

Optimisation results are detailed in Table 1 for four different time windows and the VTEC feature space, for matching VTEC along the latitudinal line segment. The log root mean square (RMS) error in VTEC for the 180 matches over each three hour window are plotted, in order of increasing RMS error, in Fig. 6. The plots illustrate that the statistical significance in the optimisation is dubious, even without factoring in the errors in the underlying SAMI2 model. Nevertheless, the optimal SAMI2 matches are plotted as dashed lines in Fig. 5. SAMI2 struggles to match the high VTEC towards the eastern coast of north America. The drift velocity model is very simple. The domain of $V$ applied in the optimisation includes values which imply velocities above the equator that are most probably physically impossible (see Appendix B). Due to dubious statistical significance, the scientific conclusions from the experiments are limited. Furthermore, the matching function is probably overly simple, the optimisation is over a coarse grid and does not involve the variation of other parameters (e.g. multiplicative factors for the neutral densities of positive ion species), and SAMI2 was run independently for each time-window, with no ability to dynamically change parameters such as $F_{10.7}$ in time. Interestingly, Jee et al. (2005) describes a sensitivity analysis for VTEC, but each parameter was varied individually and not in combination.

\section{Discussion}

Ideal or perfect image-model coupling (Smith et al., 2009) should recover the Ap and $F_{10.7}$ values exactly. Given the difficulty of the task, it is not surprising that there is significant discrepancy between the Ap values recorded in the lower graph in Fig. 1, and those obtained by the optimisation for image-model coupling as listed in Table 1. 
Table 1. Key driver variables for SAMI2 optimised against the ensemble mean from MIDAS, along the latitudinal line segment, for the VTEC feature space, and for different time windows during 20 November 2003.

\begin{tabular}{lllrrr}
\hline \multicolumn{2}{c}{ time window } & & \multicolumn{3}{c}{ optimal parameters } \\
\cline { 5 - 7 }$s$ & (UT) & & $F_{10.7}$ & Ap & $V / \mathrm{ms}^{-1}$ \\
\hline \multirow{2}{*}{1} & $12: 00-14: 00$ & & 50.0 & 0 & 225.0 \\
2 & $15: 00-17: 00$ & & 50.0 & 80 & 225.0 \\
3 & $18: 00-20: 00$ & & 300.0 & 80 & 100.0 \\
4 & $21: 00-23: 00$ & & 250.0 & 0 & 100.0 \\
\hline
\end{tabular}

SAMI2 was designed to model equatorial and low latitude processes, rather than those high latitude processes which can have a significant effect at midlatitudes during a geomagnetic storm. For example, SAMI2 was not designed to accurately model the physics of high latitude convection, and the simple sinusoidal $\boldsymbol{E} \times \boldsymbol{B}$ drift velocity model is most applicable in equatorial regions. Furthermore, SAMI2 uses the HWM93 neutral wind model. This wind model is statistical and is unlikely to adequately or accurately characterise the strong high latitude equatorward neutral winds, caused by Joule heating and electron precipitation, common during a large storm. Since the latitudinal line segment chosen for the coupling experiments is at midlatitudes where both equatorial and polar/auroral processes are likely to be influential during a geomagnetic storm, SAMI2 may be trying to replicate the build-up of plasma at midlatitudes by driving an equatorial process in an unrealistic manner. In particular, the drift velocity model may be too simple. Since variation in the parameter $V$ was used to mimic variation in electric field strength, improvements in electric field models may give better coupling results.

Ionospheric tomographic reconstruction techniques are susceptible to error (e.g. Dear and Mitchell, 2006; Pryse et al., 1998) since the problem is typically very underdetermined. As tomographic techniques and physical models improve, it may be beneficial to repeat the coupling analysis for a larger region, and to compare model output and images at timesteps more frequent than one per hour.

More fundamentally, SAMI2 was used in the "reverse direction" though it was designed to run in the "forward direction", i.e. SAMI2 was used to discriminate the most appropriate drivers given electron content in the ionosphere, rather than "generate" suitable electron content given drivers. For image-model coupling, it may be useful to introduce some techniques from discriminative learning (Duda et al., 2001) to help ionospheric models better discriminate different drivers given specific electron content.

SAMI2 models the distribution of plasma at different slices in geomagnetic longitude. There is no coupling between slices and no attempt to directly model zonal plasma

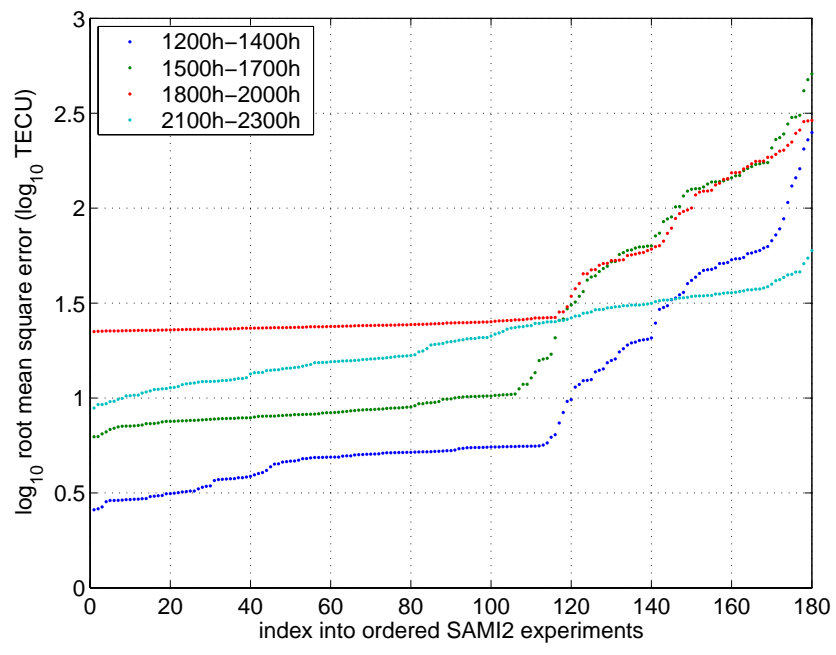

Fig. 6. Ordered sequences of log root mean square (RMS) error in VTEC between ensemble mean from MIDAS and different SAMI2 outputs, for four time windows during 20 November 2003.

drifts. Global models such as SAMI3 (SAMI3, access: September 2008) may be more appropriate. The global model should be chosen to balance accuracy with computational cost, particularly if the model has many input driver variables and any optimisation in the coupling process is likely to require many runs of the model. With high vertical $\boldsymbol{E} \times \boldsymbol{B}$ drift velocities, there is also the risk of introducing modelling innaccuracies if plasma is dragged down from the topmost field line to $1580 \mathrm{~km}$ altitude (J. D. Huba, private communication, 2008). As an example, and as detailed in Appendix C, this is possible above the geomagnetic equator for $V \geq 95.4 \mathrm{~ms}^{-1}$, though the bound is more difficult to calculate elsewhere.

As explained in Smith et al. (2009), those matching functions which account for "channel memory" and more expressive "source memory" may improve the coupling and sensitivity analysis. Also, some regularisation may be introduced which constrains the variation of key drivers between adjacent time windows. However an increase in the complexity of the matching function sometimes requires more data to estimate the accompanying statistical models robustly, or increased complexity in the search and optimisation. In general, more advanced optimisation techniques may be introduced. For example, particularly attractive are those derivative-free methods which numerically approximate the Hessian of the matching function as part of the optimisation process (Powell, 2007). The Hessian may be regarded as encoding sensitivity information at a particular instantiation of driver variables, though relative to one image only (Smith et al., 2009). There may also be benefit in augmenting the feature space with new features. 


\section{Conclusions}

Image-model coupling can be used to infer the values of driver variables which best replicate some description of electron content in the ionosphere, and ideally analyse the sensitivity of the electron content to variations in the values of those driver variables. Here, an attempt has been made to couple an ionospheric model to a tomographic reconstruction of the geomagnetic storm of 20 November 2003. A relatively simple model was chosen and few input variables were varied. The investigation confirmed the practical difficulties of this task, given that performance depends on the accuracy of the assumptions in the ionospheric model and the accuracy of the tomographic images. An ensemble technique was used to "average out" some of the variability due to different regularisation, and yield an approximate assessment of confidence in the reconstruction. The wider application of ensemble methods is recommended for analysing solutions for large underdetermined inverse problems.

\section{Appendix A}

\section{Ensemble statistics}

The $i$-th member of the ensemble is denoted by $\mathcal{M}_{i}, i \in$ $[1, n]$. For an observation $\boldsymbol{y}_{t} \in \mathbb{R}^{d_{t}}$ of uncalibrated slant TEC values at a discrete timestep $t \in[1, T]$, then $\mathcal{M}_{i}$ yields the solution,

$$
\begin{aligned}
\hat{\boldsymbol{x}}^{i}\left(\boldsymbol{y}_{t}\right) \equiv \hat{\boldsymbol{x}}^{i}\left(\boldsymbol{y}_{t} ; \check{\boldsymbol{x}}_{t}\right)= & \arg _{\boldsymbol{x}} \min \left\{f^{i}\left(\boldsymbol{y}_{t}, \boldsymbol{x}\right)\right. \\
& \left.+f_{\mathrm{reg}}^{i}\left(t, \boldsymbol{x}-\check{\boldsymbol{x}}_{t}\right)\right\},
\end{aligned}
$$

where the sum square error term is,

$$
\begin{aligned}
& f^{i}\left(\boldsymbol{y}_{t}, \boldsymbol{x}\right)=\left\|\boldsymbol{e}^{i}\left(\boldsymbol{y}_{t}, \boldsymbol{x}\right)\right\|_{\mathbf{A}_{t}^{-1}}^{2}=\left[\boldsymbol{e}^{i}\left(\boldsymbol{y}_{t}, \boldsymbol{x}\right)\right]^{\top} \mathbf{A}_{t}^{-1} \boldsymbol{e}^{i}\left(\boldsymbol{y}_{t}, \boldsymbol{x}\right), \\
& \boldsymbol{e}^{i}\left(\boldsymbol{y}_{t}, \boldsymbol{x}\right)=\boldsymbol{y}_{t}-\left[\mathbf{H}_{t} \vdots \mathbf{B}_{t}\right]\left[\begin{array}{c}
\boldsymbol{x} \\
\boldsymbol{b}(\boldsymbol{x})
\end{array}\right]
\end{aligned}
$$

the regularisation term is,

$$
\begin{aligned}
f_{\text {reg }}^{i}(t, \boldsymbol{x})= & \alpha_{1}^{t}\left(\lambda_{1}^{i}\right) \sum_{j \in \mathcal{J}}\|\boldsymbol{x}\|_{\mathrm{L} 2}^{2}+\alpha_{2}^{t}\left(\lambda_{2}^{i}\right) \sum_{j \in \mathcal{J}}\left\|\nabla_{j}[\boldsymbol{x}]\right\|_{\mathrm{L} 2}^{2} \\
& +\alpha_{3}^{t}\left(\lambda_{3}^{i}\right) \sum_{j \in \mathcal{J}}\left\|\nabla_{j}\left[\left(\nabla_{j}[\boldsymbol{x}]\right)^{\top}\right]\right\|_{\mathrm{L} 2}^{2},
\end{aligned}
$$

and the implicit noise term $\boldsymbol{n}_{t}$ is drawn from a continuous distribution,

$\boldsymbol{n}_{t} \sim \mathcal{N}\left(\mathbf{0}, \mathbf{A}_{t}\right)$.

Here $x$ denotes a vector of electron density values across the relevant grid, with implicit prior reference $\breve{\boldsymbol{x}}_{t}$ at time $t$; $\mathbf{H}_{t}$ and $\mathbf{B}_{t}$ are the projection matrix and transmitter/receiver offset indicator matrix both at time $t ; \boldsymbol{x} \mapsto \boldsymbol{b}$ is assumed injective according to a least squares solver; $\left(\lambda_{1}^{i}, \lambda_{2}^{i}, \lambda_{3}^{i}\right)$ is a unique set of regularisation parameter values where $\lambda_{k}^{i} \in$ $\mathbb{R}_{0}^{+}, k \in\{1,2,3\} ; \nabla_{j}$ denotes the first-order derivative operator in direction $j$, " $\top$ " the transpose operator, and $\mathcal{N}(\cdot, \cdot)$ a continuous Gaussian distribution with mean and covariance as its first and second arguments respectively. For $\lambda \geq 0$, each $\alpha_{k}^{t}(\lambda) \geq 0$ is a time-dependent scalar function.

In the experiments, the index $j$ denoting derivative directions was drawn from a set $\mathcal{J}$ of four members describing directions of increasing latitude, increasing longitude, increasing latitude/increasing longitude, and decreasing latitude/increasing longitude. The first-order derivative was based on the basis $(-1,0,1)$ which was scaled to maintain a fixed gradient in all directions in the space of cell indices, not in the space of absolute distances. A similar remark follows for the second-order derivatives except that the basis was $(1,-2,1)$. Also, in the experiments the noise covariance was $\boldsymbol{A}_{t}=a_{t} \mathbf{I}$, where $\mathbf{I}$ is the Identity matrix. Then $\alpha_{k}^{t}\left(\lambda_{k}^{i}\right)=\left(1 / a_{t}\right) c_{k}^{t} \lambda_{k}^{i}$ where $c_{k}^{t} \in \mathbb{R}_{0}^{+}$is independent of model $\mathcal{M}_{i}$ and was determined by entries into the quadratic programming solver. As a result, each $\lambda_{k}^{i}$ may simply be regarded as a scaling parameter for the relevant regularisation term.

Given a function $F: \boldsymbol{y}_{t} \mapsto F\left(\boldsymbol{y}_{t}\right)$ and assuming $\left(\boldsymbol{y}_{t}, \mathcal{M}_{i}\right) \mapsto \hat{\boldsymbol{x}}^{i}\left(\boldsymbol{y}_{t}\right)$ is injective, then with slight abuse of notation, posterior averaging yields,

$$
\begin{aligned}
F\left(\boldsymbol{y}_{t}\right) & =\sum_{i=1}^{n} F\left(\boldsymbol{y}_{t}, \mathcal{M}_{i}\right) P\left(\mathcal{M}_{i} \mid \boldsymbol{y}_{t}\right), \\
& =\sum_{i=1}^{n} F\left(\hat{\boldsymbol{x}}^{i}\left(\boldsymbol{y}_{t}\right)\right) P\left(\mathcal{M}_{i} \mid \boldsymbol{y}_{t}\right),
\end{aligned}
$$

where $P(\cdot)$ denotes a probability mass function. Assuming member priors are equal, then the member posterior may be simplified as follows,

$$
\begin{aligned}
P\left(\mathcal{M}_{i} \mid \tilde{\boldsymbol{y}}_{t}\right) & =\frac{p\left(\tilde{\boldsymbol{y}}_{t} \mid \mathcal{M}_{i}\right) P\left(\mathcal{M}_{i}\right)}{\sum_{j=1}^{n} p\left(\tilde{\boldsymbol{y}}_{t} \mid \mathcal{M}_{j}\right) P\left(\mathcal{M}_{j}\right)}, \\
& =\frac{p\left(\tilde{\boldsymbol{y}}_{t} \mid \hat{\boldsymbol{x}}^{i}\left(\boldsymbol{y}_{t}\right)\right)}{\sum_{j=1}^{n} p\left(\tilde{\boldsymbol{y}}_{t} \mid \hat{\boldsymbol{x}}^{j}\left(\boldsymbol{y}_{t}\right)\right)}, \\
& =\frac{\exp \left\{-(1 / 2) f^{i}\left(\tilde{\boldsymbol{y}}_{t}, \hat{\boldsymbol{x}}^{i}\left(\boldsymbol{y}_{t}\right)\right)\right\}}{\sum_{j=1}^{n} \exp \left\{-(1 / 2) f^{j}\left(\tilde{\boldsymbol{y}}_{t}, \hat{\boldsymbol{x}}^{j}\left(\boldsymbol{y}_{t}\right)\right)\right\}}, \\
& \equiv w^{i}\left(\tilde{\boldsymbol{y}}_{t}\right) .
\end{aligned}
$$

where the likelihood probability density function $p\left(\tilde{\boldsymbol{y}}_{t} \mid \hat{\boldsymbol{x}}^{i}\left(\boldsymbol{y}_{t}\right)\right)$ is a Gaussian distribution, and held-out test data is denoted by observations $\tilde{\boldsymbol{y}}_{t} \in \mathbb{R}^{\tilde{d}_{t}}, t \in[1, T]$. Then $a_{t}$ can be estimated using the held-out data,

$a_{t}=\frac{1}{n \tilde{d}_{t}} \sum_{i=1}^{n}\left\|\boldsymbol{e}^{i}\left(\tilde{\boldsymbol{y}}_{t}, \hat{\boldsymbol{x}}^{i}\left(\boldsymbol{y}_{t}\right)\right)\right\|_{\mathrm{L} 2}^{2}$. 
The above analysis is consistent with treating each timestep separately. An arithmetic average for posterior weights across timesteps may be taken to encourage robustness. For example,

$\tilde{w}^{i}=\frac{1}{T} \sum_{\tau=1}^{T} w^{i}\left(\tilde{\boldsymbol{y}}_{\tau}\right)$

Then,

$F\left(\boldsymbol{y}_{t}\right)=\sum_{i=1}^{n} \tilde{w}^{i} F\left(\hat{\boldsymbol{x}}^{i}\left(\boldsymbol{y}_{t}\right)\right)$

An example of $F$ is the operator extracting the VTEC. Since it is often useful to give some indication of confidence in the point estimate, assume VTEC $z_{t} \sim \mathcal{N}\left(\boldsymbol{\mu}_{t}, \boldsymbol{\Sigma}_{t}\right)$, abbreviate $z_{t}^{i} \equiv z\left(\hat{\boldsymbol{x}}^{i}\left(\boldsymbol{y}_{t}\right)\right)$ and let,

$\boldsymbol{\mu}_{t}=\sum_{i=1}^{n} \tilde{w}^{i} z_{t}^{i}$

$\boldsymbol{\Sigma}_{t}=\frac{1}{n} \sum_{i=1}^{n} \tilde{w}^{i}\left(z_{t}^{i}-\boldsymbol{\mu}_{t}\right)\left(z_{t}^{i}-\boldsymbol{\mu}_{t}\right)^{\top}$,

The 95\% confidence intervals for the $k$-th element of $z_{t}$ occur at $\mu_{t}^{k} \pm 1.96 \sigma_{t}^{k}$ (Korn and Korn, 1968), where $\mu_{t}^{k}$ is the $k$-th element of $\boldsymbol{\mu}_{t}, \sigma_{t}^{k}=\sqrt{\Sigma_{t}(k, k)} \geq 0$ and $\Sigma_{t}(k, k)$ is the $(k, k)$-th element of $\boldsymbol{\Sigma}_{t}$.

\section{Appendix B}

\section{The $E \times B$ sinusoidal drift velocity model}

The vertical component of the $\boldsymbol{E} \times \boldsymbol{B}$ drift velocity, according to the "sinusoidal model" in Huba et al. (2000), varies as the following scalar field. Expressing all angles in radians, unless otherwise stated, and defining a critical altitude $h_{\text {crit }}$, then the vertical component at altitude $h \geq h_{\text {crit }}$, geomagnetic latitude $\theta \in[-\pi / 2, \pi / 2]$ and local time $t$ in hours is,

$v(h, \theta, t)=V(h, \theta) \sin \left(\frac{\pi(t-7)}{12}\right)$,

where,

$V(h, \theta)=V \cos (\alpha(h, \theta)) \frac{\cos ^{3}(\theta)}{\left(1+3 \sin ^{2}(\theta)\right)^{1 / 2}} \frac{(h+R)^{2}}{R^{2}}$,

and where $\alpha(h, \theta)$ is the angle the magnetic field line at $(h, \theta)$ makes with the local horizontal, $R$ is the radius of the Earth (in the same units as altitude), and $V$ is the parameter quoted in the investigations. For $h<h_{\text {crit }}$, the term $V(h, \theta)$ decays exponentially. Hence $V$ is simply a mathematical parameter which may be interpreted as the, usually hypothetical, peak
$\boldsymbol{E} \times \boldsymbol{B}$ drift velocity at zero altitude at the geomagnetic equator, where such drift is strictly vertical. According to this model, the localised peak vertical drift velocity $V(h, \theta)$ increases quadratically with increasing altitude, but decreases northwards and southwards of the geomagnetic equator due to the convergence of field lines and their dipping relative to the local horizontal. As a result, the actual vertical $\boldsymbol{E} \times \boldsymbol{B}$ drift velocities at northerly latitudes are much less than those at the geomagnetic equator. For example, within the north America region used in the investigations between $90 \mathrm{~km}$ and $1580 \mathrm{~km}$ inclusive, approximate calculations gave the localised peak vertical drift velocity $V(h, \theta)$ as varying between $0.49 \mathrm{~V}$ and $0.07 \mathrm{~V}$ near $20^{\circ} \mathrm{N}$ and $40^{\circ} \mathrm{N}$ geographic latitude respectively, with an average of $0.20 \mathrm{~V}$. For reference, between the same altitude limits and along the lines of geomagnetic longitude used in the experiments detailed above, the maximum vertical component of localised peak $\boldsymbol{E} \times \boldsymbol{B}$ drift velocity was approximated at $1.50 \mathrm{~V}$ near the geomagnetic equator.

\section{Appendix C}

\section{Calculating plasma displacement due to $E \times B$ drift}

At the geomagnetic equator plasma displacement is in the radial direction so that, applying the $\boldsymbol{E} \times \boldsymbol{B}$ drift velocity model described in Appendix B,

$\frac{\mathrm{d} h}{\mathrm{~d} t^{\prime}}=V^{\prime} \frac{(h+R)^{2}}{R^{2}} \sin \left(t^{\prime}\right)$,

where $t^{\prime}=\pi(t-7) / 12, t^{\prime}$ is dimensionless and $t$ is in hours, and where $V^{\prime}$ is expressed in units consistent with $t^{\prime}$ and altitude $h$ such that the velocity $V$ in $\mathrm{ms}^{-1}$ is,

$V=\frac{1000 \cdot \pi}{12 \cdot 60^{2}} V^{\prime}$.

Between $t_{1}^{\prime}$ and $t_{2}^{\prime}$, assume it is physically possible to displace plasma from $h_{1}$ to $h_{2}$. Solving the ordinary differential equation (Korn and Korn, 1968),

$\int_{h_{1}}^{h_{2}} \frac{R^{2}}{(h+R)^{2} V^{\prime}} \mathrm{d} h=\int_{t_{1}^{\prime}}^{t_{2}^{\prime}} \sin \left(t^{\prime}\right) \mathrm{d} t^{\prime}$.

Of interest is the velocity $V^{\prime}$ required to drag plasma down from $h_{1}$ to $h_{2}$ over half a day between $t_{1}^{\prime}=\pi$ and $t_{2}^{\prime}=2 \pi$,

$V^{\prime}=\frac{R^{2}}{2}\left(\frac{1}{\left(h_{2}+R\right)}-\frac{1}{\left(h_{1}+R\right)}\right)$.

Relating this to the investigations reported above, then when $h_{1}=10000 \mathrm{~km}, h_{2}=1580 \mathrm{~km}$ and $R=6365 \mathrm{~km}$, then $V=95.4 \mathrm{~ms}^{-1}$. This implies that when the parameter $V \geq$ $95.4 \mathrm{~ms}^{-1}$, plasma is dragged down in the $\boldsymbol{E} \times \boldsymbol{B}$ direction 
from the upper field line above the geomagnetic equator to the top of the grid structure at an altitude of $1580 \mathrm{~km}$. However it is more difficult to calculate the velocity parameter $V$ which is required to potentially drag down plasma from above nonzero geomagnetic latitudes. Although the topmost field line has a lower altitude above such latitudes, the relevant peak $\boldsymbol{E} \times \boldsymbol{B}$ drift velocity there, according to the model, is also lower.

Acknowledgements. N. D. Smith and C. J. Budd were supported in BICS by EPSRC grant GR/S86525/01. For financial support, D. Pokhotelov would like to thank the STFC, and C. N. Mitchell the STFC and EPSRC. The authors would also like to thank Paul Spencer for his work in developing MIDAS which formed the basis for these experiments including the regularised least-squares methodology, and for useful discussion; the Naval Research Laboratory for providing SAMI2, and J. D. Huba for helpful comments on SAMI2; the providers of IRI-95 (Bilitza, 1997); the providers of OMNI data via the GSFC/SPDF OMNIWeb interface, including N. F. Ness as PI for the ACE IMF data (see OMNIWeb, access: July 2010 for relevant details including contributors); the International GNSS Service (IGS) ${ }^{1}$ for the IGS data (Dow et al., 2009), and the Scripps Orbit and Permanent Array Center (SOPAC $)^{3}$ for making the IGS data available via the SOPAC/CSRC Archive at http://garner.ucsd.edu; and the referees for helpful comments, particularly regarding the need to assess confidence. The investigation used MATLAB ${ }^{2}$.

Edited by: J. Kurths

Reviewed by: two anonymous referees

\section{References}

Bhuyan, K. and Bhuyan, P.: International Reference Ionosphere as a potential regularization profile for computerized ionospheric tomography, Adv. Space Res., 39, 851-858, 2007.

Bilitza, D.: International Reference Ionosphere - Status 1995/96, Adv. Space Res., 20, 1751-1754, 1997.

Bust, G. and Mitchell, C.: History, current state, and future directions of ionospheric imaging, Rev. Geophys., 46, RG1003, doi:10.1029/2006RG000212, 2008.

Dear, R. and Mitchell, C.: GPS interfrequency biases and total electron content errors in ionospheric imaging over Europe, Radio Sci., 41, RS6007, doi:10.1029/2005RS003269, 2006.

Dow, J., Neilan, R., and Rizos, C.: The International GNSS Service in a changing landscape of Global Navigation Satellite Systems, J. Geodesy, 83, 191-198, 2009.

Duda, R., Hart, P., and Stork, D.: Pattern Classification, 2nd edn., A Wiley-Interscience Publication, John Wiley \& Sons, Inc., New York, 2001.

Foster, J., Coster, A., Erickson, P., Rideout, W., Rich, F., Immel, T., and Sandel, B.: Redistribution of the Stormtime Ionosphere and the Formation of a Plasmaspheric Bulge, in: Inner Magnetosphere Interactions: New Perspectives from Imaging, edited by: Burch, J., Schulz, M., and Spence, H., American Geophysical
Union, Washington, DC, Geoph. Monog. Series, 159, 277-289, 2005.

Hargreaves, J.: The solar-terrestrial environment, in: Cambridge atmospheric and space science series, Cambridge University Press, Cambridge, 2003.

Huba, J., Joyce, G., and Fedder, J.: Sami2 is Another Model of the Ionosphere (SAMI2): A new low-latitude ionosphere model, J. Geophys. Res., 105, 23035-23053, 2000.

Jee, G., Schunk, R., and Scherliess, L.: On the sensitivity of total electron content (TEC) to upper atmospheric/ionospheric parameters, J. Atmos. Sol.-Terr. Phy., 67, 1040-1052, doi: 10.1016/j.jastp.2005.04.001, 2005.

Korn, G. and Korn, T.: Mathematical Handbook for Scientists and Engineers: Definitions, Theorems, and Formulas for Reference and Review, 2nd, enlarged and revised edn., McGraw-Hill, Inc., New York, 1968.

Mannucci, A., Tsurutani, B., Iijima, B., Komjathy, A., Wilson, B., Pi, X., Sparks, L., Hajj, G., Mandrake, L., Gonzalez, W., Kozyra, J., Yumoto, K., Swisdak, M., Huba, J., and Skoug, R.: Hemispheric Daytime Ionospheric Response To Intense Solar Wind Forcing, in: Inner Magnetosphere Interactions: New Perspectives from Imaging, edited by: Burch, J., Schulz, M., and Spence, H., American Geophysical Union, Washington, DC, Geoph. Monog. Series, 159, 261-275, 2005.

Mitchell, C. and Spencer, P.: A three-dimensional time-dependent algorithm for ionospheric imaging using GPS, Ann. Geophys.Italy, 46, 687-696, 2003.

OMNIWeb: Space Physics Data Facility, NASA/Goddard Space Flight Center, available at: http://omniweb.gsfc.nasa.gov, last access: July 2010.

Powell, M.: A view of algorithms for optimization without derivatives, Mathematics TODAY, 43, 170-174, 2007.

Pryse, S., Kersley, L., Mitchell, C., Spencer, P., and Williams, M.: A comparison of reconstruction techniques used in ionospheric tomography, Radio Sci., 33, 1767-1779, 1998.

SAMI2: The SAMI2 Open Source Project, Naval Research Laboratory, available at: http://wwwppd.nrl.navy.mil/sami2-OSP/ index.html, last access: November 2007.

SAMI3: NRL Ionosphere Model: SAMI3, available at: http: //www.nrl.navy.mil/content.php?P=04REVIEW105, last access: September 2008.

Smith, N. D., Mitchell, C. N., and Budd, C. J.: Image-model coupling: a simple information theoretic perspective for image sequences, Nonlin. Processes Geophys., 16, 197-210, doi:10.5194/npg-16-197-2009, 2009.

Spencer, P. and Mitchell, C.: Imaging of fast moving electrondensity structures in the polar cap, Ann. Geophys.-Italy, 50, 427434, 2007.

Tsurutani, B. T., Verkhoglyadova, O. P., Mannucci, A. J., Araki, T., Sato, A., Tsuda, T., and Yumoto, K.: Oxygen ion uplift and satellite drag effects during the 30 October 2003 daytime superfountain event, Ann. Geophys., 25, 569-574, doi:10.5194/angeo25-569-2007, 2007.

\footnotetext{
${ }^{3}$ http://sopac.ucsd.edu
} 